\title{
Thermal, Caloric and Transport Properties of the Lennard-Jones Truncated and Shifted Fluid in the Adsorbed Layers at Dispersive Solid Walls ${ }^{1}$
}

\author{
Martin P. Lautenschlaeger, Hans Hasse \\ Laboratory of Engineering Thermodynamics (LTD), University of Kaiserslautern, \\ Erwin-Schrödinger-Straße 44, 67663 Kaiserslautern, Germany
}

\begin{abstract}
Fluid properties change when the fluid is adsorbed at a wall. The effect of the adsorption on the fluid properties was studied here by molecular simulation. There is much previous work in this field on fluids in nanochannels that are so small that the adsorbed layers on both walls interfere. In the present work the channel width was so large that the adsorbed layers did not interfere, such that information on the adsorbed layer on single walls was obtained and average values of thermodynamic properties of the fluid in that layer were determined. The studied fluid properties are: pressure $p$, density $\rho$, internal energy $u$, enthalpy $h$, isobaric heat capacity $c_{\mathrm{p}}$, thermal expansion coefficient $\alpha_{\mathrm{p}}$, thermal conductivity $\lambda$, shear viscosity $\eta$, and self-diffusion coefficient $D$. For the study, non-equilibrium molecular dynamics simulations were carried out, i.e. gradients of the temperature and the velocity along different directions were applied. The fluid and the solid were modeled with the Lennard-Jones potential truncated and shifted at $r_{\mathrm{c}}^{*}=2.5 \sigma$. The overall density of the fluid was always $\bar{\rho}=0.8$ in the present simulations. The overall temperature as well as the solid-fluid interaction were varied. The corresponding bulk states are liquid or supercritical. The results for the fluid properties in the adsorbed layer were compared to the corresponding bulk values and the deviations were found to be generally below $15 \%$. Keywords: adsorption; solid-fluid interface; wetting; anisotropy
\end{abstract}

\footnotetext{
${ }^{1}$ The Version of Record of this manuscript has been published and is available in MOLECULAR PHYSICS 23 Sep 2019 DOI:10.1080/00268976.2019.1669838
} 


\section{Introduction}

The behavior of fluids at solid-fluid interfaces plays an important role in many technical and natural processes such as heat transfer, tribology, coating, and adhesion. Molecular dynamics (MD) simulations are suited to study the behavior of fluids at such interfaces [1-4]. Using MD simulations, e.g., it has been found that upon adsorption at the solid surface, the fluid develops a layered structure. In simple fluids as they are also studied in the present work, this structure can be observed typically up to a distance of approximately five molecular diameters away from the solid-fluid interface [1, 5] 7]. Within this region fluid properties and the phase behavior differ from that of the bulk fluid [1, 5, 8 11]. At a larger distance the structural effects vanish and the fluid properties approach to their bulk values.

Many authors have studied the structure of these adsorbed layers, e.g. References [6, 8, 12 14. Less is known on thermodynamic and transport properties of the fluid in the adsorbed layers [15]. MD simulations have been used before to study the following properties of simple fluids adsorbed to walls: self-diffusivity [8, 15, 24], thermal conductivity [23, 25, 29], and shear viscosity [23, 29, 30]. In most of these studies, fluid properties were determined as mean values in nanochannels that were so small that the adsorbed layers on both walls interfered [1, 5, 6, 13, 16, 31, 32. Then, the height of the channel determines the structure of the fluid, which is sometimes called frustration of ordering [12, 33]. Small changes in the height may then lead to important changes of the ordering. In such situations, the height of the channel has a strong influence on the properties of the fluid in the channel [11].

The present study is concerned only with situations in which frustration of ordering plays no role: in principle a simple fluid at an atomically structured flat wall is considered. The features that distinguish the present study are: (1) The simulation setup corresponds to a nanochannel with a height of approximately 20 molecular diameters such that the adsorbed layers from the opposite walls do not interfere. Structural effects due to confinement, i.e. frustration of ordering, are avoided. They are solely induced by adsorption. (2) Fluid properties are reported as mean values for the five fluid layers that are closest to the solid-fluid interface and not as mean values for the entire nanochannel. Thus, 
the reported results are averages for the region in which adsorption leads to an atomistic layering structure and do not include contributions from the bulk. (3) The solid-fluid interaction is specified in such a way that it yields a realistic wetting behavior of the solid, which covers also the limiting cases full wetting and full dewetting, see Reference [34]. (4) Not only the strength of the solid-fluid interaction is varied in a wide range but also the temperature. I.e. on a molecular level the ratio of the kinetic energy and the potential energy of adsorption is varied systematically.

Non-equilibrium molecular dynamics (NEMD) simulations were carried out. The studied simulation system consists only of Lennard-Jones truncated and shifted sites with a cut-off radius of $r_{\mathrm{c}}^{*}=2.5 \sigma$ (LJTS). An atomically structured solid LJTS wall is in contact with a LJTS fluid. The overall fluid density was $\bar{\rho}=0.8$ in all simulations. The overall temperature was varied: $\bar{T}=\{0.7,0.8,2.0\}$. The solid-fluid interaction $\varepsilon_{\text {sf }}$ was varied in the range of $\varepsilon_{\mathrm{sf}}=[0.25,0.75]$ which according to Becker et al. [34] corresponds to the contact angles $\phi=[0, \pi]$. The properties of the adsorbed LJTS fluid that were studied in the present work are: pressure $p$, density $\rho$, internal energy $u$, enthalpy $h$, isobaric heat capacity $c_{\mathrm{p}}$, thermal expansion coefficient $\alpha_{\mathrm{p}}$, thermal conductivity $\lambda$, shear viscosity $\eta$, and self-diffusion coefficient $D$. The thermal conductivity was studied along the direction normal to the solid-fluid interface, i.e. $\lambda_{\perp}$, and parallel to the solid-fluid interface, i.e. $\lambda_{\|}$, and behaves anisotropic. To determine the properties $c_{\mathrm{p}}, \alpha_{\mathrm{p}}, \lambda_{\perp}, \lambda_{\|}$, and $\eta$ using NEMD, gradients of the temperature and the velocity along different directions are applied. Therefore, in the present work three variants of the simulation setup that differ in the number and position of the temperature and velocity control volumes are used. For a given density, the average numbers of the aforementioned fluid properties in the interfacial region are reported as a function of the temperature and the solid-fluid interaction and the results are compared with the corresponding bulk values. Deviations are found for most of the fluid properties. As expected, they depend strongly on $\varepsilon_{\mathrm{sf}}$. Moreover, a significant effect of the temperature on these deviations is found for some properties.

This paper is organized as follows: the LJTS force field is specified in Section 2. In Section 3 information on the simulations is given. This includes the description of the simulation setup, the determination of the different fluid properties as well as the error analysis. The results are presented and discussed in Section 4 . Finally, the conclusions 
are drawn in Section 5 .

\section{Molecular Model}

The studied system consists of two components: a solid wall (index 's') and a fluid (index ' $\mathrm{f}$ '). The Lennard-Jones potential truncated and shifted at the cut-off radius $r_{\mathrm{c}}^{*}=$ $2.5 \sigma$ is used to describe all molecular interactions, i.e. those between sites of the same type (index 'ff' or 'ss') as well as those between sites of different types (index 'sf').

$$
u^{\mathrm{LJTS}}\left(r_{i j}\right)=\left\{\begin{array}{ll}
u^{\mathrm{LJ}}\left(r_{i j}\right)-u^{\mathrm{LJ}}\left(r_{\mathrm{c}}\right), & r_{i j}<r_{\mathrm{c}} \\
0, & r_{i j} \geq r_{\mathrm{c}}
\end{array},\right.
$$

where

$$
u^{\mathrm{LJ}}\left(r_{i j}\right)=4 \varepsilon\left[\left(\sigma / r_{i j}\right)^{12}-\left(\sigma / r_{i j}\right)^{6}\right]
$$

$\varepsilon$ is the energy parameter, describing the dispersive attraction and $\sigma$ is the size parameter describing the repulsion. $r_{i j}$ is the distance between the two LJTS particles $i$ and $j$. The LJTS potential is well suited for describing simple nonpolar fluids [35]. This includes the interfacial properties of the fluid in which we are particularly interested in the present work [34, 36]. However, the LJTS model gives only crude descriptions of properties of solids. As these are not of particular interest here, the simple LJTS model is also used for modeling the solid to keep the simulations simple and efficient.

The size parameter $\sigma_{\mathrm{f}}$ is used to normalize all distances. Its value is identical for all LJTS sites. All energies are normalized using the LJTS energy parameter of the fluid $\varepsilon_{\mathrm{f}}$. The cohesive energy of the solid is set to $\varepsilon_{\mathrm{s}}=100 \varepsilon_{\mathrm{f}}$. The energy parameter of the solid-fluid interaction is varied in the range $\varepsilon_{\mathrm{sf}}=[0.25,0.75] \varepsilon_{\mathrm{f}}$. According to Becker et al. [34] this corresponds to contact angles in the range $\phi=[0, \pi]$.

In the following all observables are given in reduced units which are reduced with respect to the LJTS parameters of the fluid, cf. Table 1.

\section{Simulation}

\subsection{Simulation Setup}

The simulation scenario is schematically shown in Figure 1. It is used in different ways that are explained in the subsequent sections. The general scenario consists of a wall in the 
Table 1: Definition of the physical quantities in reduced units (plain). The corresponding observable carrying a dimension is marked by the asterisk and reduced by the LJTS parameters of the fluid, i.e. $\varepsilon_{\mathrm{f}}=1, \sigma_{\mathrm{f}}=1$ and $M_{\mathrm{f}}=1$. For simplicity the index ' $\mathrm{f}$ ' is omitted in this table. $k_{\mathrm{B}}$ is the Boltzmann's constant.

\begin{tabular}{lr}
\hline Length & $L=\frac{L^{*}}{\sigma}$ \\
Time & $\tau=\frac{\tau^{*}}{\sigma \sqrt{M / \varepsilon}}$ \\
Mass & $m=\frac{m^{*}}{M}$ \\
Velocity & $\boldsymbol{v}_{k}=\frac{\boldsymbol{v}_{k}^{*}}{\sqrt{\varepsilon / M}}$ \\
Shear rate & $\dot{\gamma}=\frac{\dot{\gamma}^{*}}{\sqrt{\varepsilon / M} / \sigma}$ \\
Density & $\rho=\rho^{*} \sigma^{3}$ \\
Temperature & $T=\frac{T^{*}}{\varepsilon / k_{\mathrm{B}}}$ \\
Stresses & $\boldsymbol{\sigma}_{k l}=\frac{\boldsymbol{\sigma}_{k l}^{*}}{\varepsilon / \sigma^{3}}$ \\
Energy flux & $\boldsymbol{j}_{k}=\frac{\boldsymbol{j}_{k}^{*}}{\varepsilon \sqrt{\varepsilon / M} / \sigma^{3}}$ \\
Internal energy & $u=\frac{u^{*}}{\varepsilon}$ \\
Enthalpy & $h=\frac{h^{*}}{\varepsilon}$ \\
Thermal conductivity & $\lambda=\frac{\lambda^{*}}{k_{\mathrm{B}} \sqrt{\varepsilon / M} / \sigma^{2}}$ \\
Shear viscosity & $\eta=\frac{\eta^{*}}{\sqrt{\varepsilon M / \sigma^{2}}}$ \\
Self-diffusion coefficient & $D=\frac{D^{*}}{\sigma \sqrt{\varepsilon / M}}$ \\
Isobaric heat capacity & $c_{\mathrm{p}}=\frac{c_{\mathrm{p}}^{*}}{k_{\mathrm{B}}}$ \\
Thermal expansion coefficient & $\alpha_{\mathrm{p}}=\frac{\alpha_{\mathrm{p}}^{*}}{k_{\mathrm{B}} / \varepsilon}$ \\
\hline
\end{tabular}

$x z$-plane which is submersed in a fluid. The total number of particles is about 5,200 , from which about 1,400 particles are wall particles. The rest are fluid particles. The simulation box has the dimensions $L_{x}=15.5, L_{y}=25.7$, and $L_{z}=15.5$. This is large enough to avoid finite-size effects, especially in the regions near the wall, where the structuring due to the adsorption occurs and where interfacial features dominate the fluid behavior [37, 38]. The center of the wall in $y$-direction is fixed at $y=0$. The wall consists of seven layers of individual atoms that are arranged in a face-centered cubic (fcc) lattice with the lattice constant $a=1.55$. In the wall, the distance from the center of the bottom layer to the center of the top layer in $y$-direction is $3 a$. It exceeds the cut-off radius such that 
artifacts from the interaction of fluid particles on different sides of the wall are avoided. The thickness of the wall is defined as $H=3 a+2 \cdot 0.5=5.65$, where the contribution $2 \cdot 0.5$ considers the radii of the wall's surface particles. The length $L$ and the width $W$ of the wall are identical to the system dimensions, i.e. $L=L_{x}$ and $W=L_{z}$, respectively. At the upper and the lower surface of the wall the (100) surface orientation is exposed to the fluid. Periodic boundary conditions are applied in all directions such that the setup corresponds to a channel with the height $d=L_{y}-H \approx 20$.

Apart from the wall volume $(L \times H \times W)$, fluid particles can access the entire simulation box, cf. Figure 1. In the accessible region, the overall density of the fluid is $\bar{\rho}=0.8$ throughout the present work and the overall temperature of the fluid is varied in the range of $\bar{T}=\{0.7,0.8,2.0\}$. As schematically shown in Figure 1 , the entire fluid region is divided into interfacial regions in which data are taken (hatched areas), and bulk fluid regions (grey-shaded areas). In addition, there are regions (plain areas) where either temperature or velocity control volumes are applied optionally, cf. Figure 2, Thus, close to the solid-fluid interface a specific temperature can be applied to fluid particles between the first minimum and the first maximum of the density profile, cf. Figure 1 (left). The interfacial regions are defined here such that they include the range from the first maximum to the sixth minimum of the density profile along the $y$-direction. The $y$-position of these extrema may slightly vary with the magnitude of $\varepsilon_{\mathrm{sf}}$ and $\bar{T}$. Thus, in the present work the boundaries of the interfacial region in $y$-direction are adjusted for each simulation run separately. The thickness of the interfacial region is $H_{\mathrm{ir}}=3.8$. Within the interfacial regions values for all aforementioned fluid properties are determined as averages. In the bulk fluid regions, which are not affected by the layering structure, the mean values of density and temperature agree largely with the corresponding overall values. The deviations are extremely small and always below $0.2 \%$ for all simulations of the present work.

The mean temperature of the wall is equal to $\bar{T}$ in all simulations. It is actively controlled by applying a Gaussian isokinetic thermostat to all wall particles. The temperature of the fluid is also controlled in some regions using Gaussian isokinetic thermostats. Details concerning the location of the control volumes and the target temperatures are given below.

The interfacial region is uniformly discretized in $x$ - and $y$-direction so that bins with 


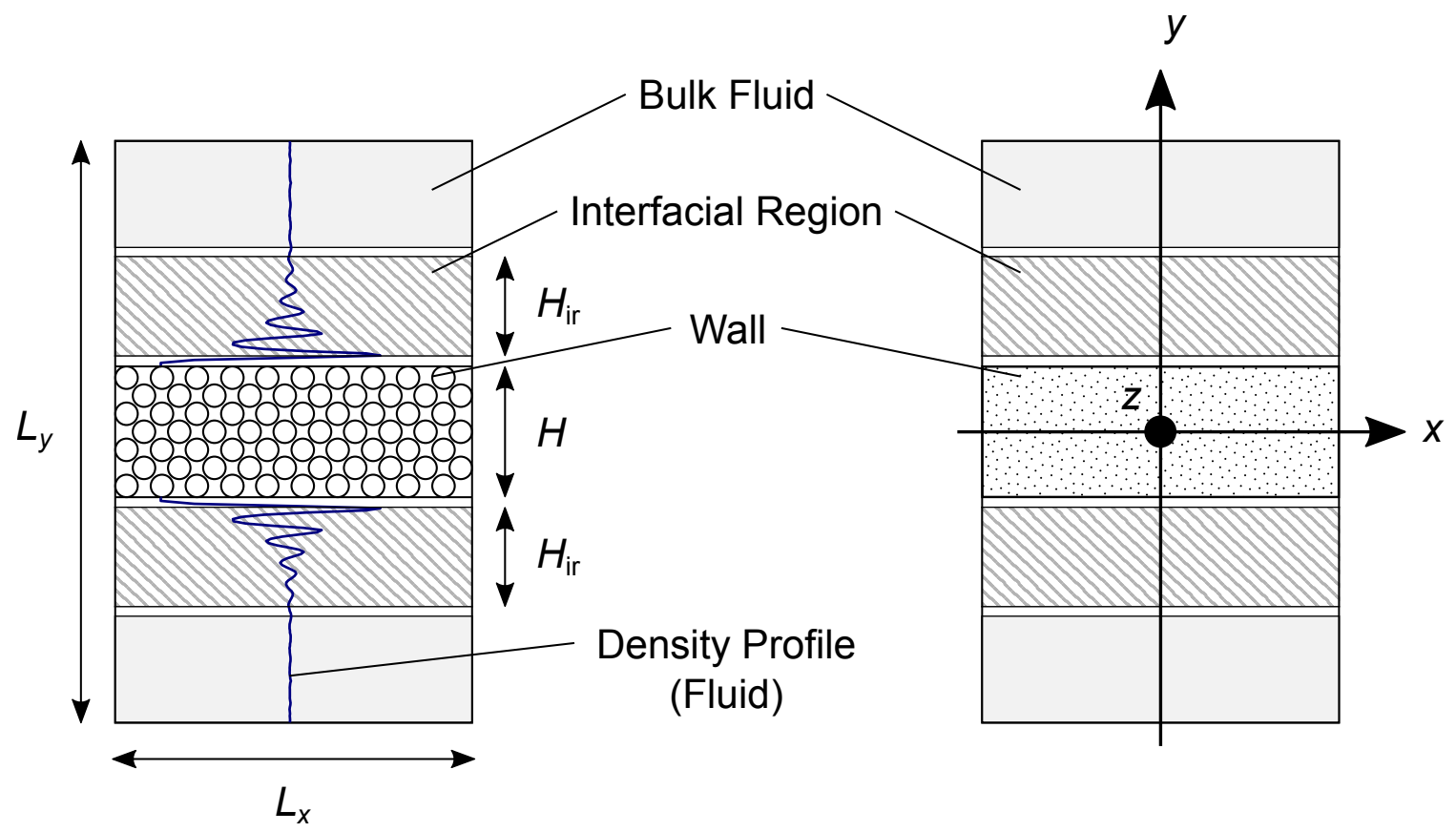

Figure 1: General simulation setup. Both schemes represent the same physical scenario and are symmetrically identical to the $x z$-plane at $y=0$ and to the $y z$-plane at $x=0$. Left: The dimensions of the system along the $x$ - and $y$-direction are $L_{x}$ and $L_{y}$, respectively. A wall with thickness $H$ is submersed in a fluid. The wall consists of individual atoms depicted with empty circles. Schematic density profiles of the fluid are shown as blue lines. They indicate a structural influence of the fluid up to the fifth layer away from the solid-fluid interface. The height of the interfacial region (hatched area) is $H_{\mathrm{ir}}$. The bulk fluid regions (grey-shaded area) are not affected by the layering structure. In the plain regions either temperature or velocity control volumes are applied optionally. Right: The wall is depicted as dotted area and the coordinate system is introduced. 
equal dimensions are obtained. The size of a bin in $x^{-}, y^{-}$, and $z$-direction is $\Delta x=0.775$, $\Delta y=0.1$, and $\Delta z=L_{z}$, respectively. Within each bin the scalar properties number density $\rho$, pressure $p$, temperature $T$, internal energy $u$, enthalpy $h$, the vectors velocity $\boldsymbol{v}$, heat flux $\boldsymbol{j}$, and self-diffusivity $\boldsymbol{D}$, as well as the stress tensor $\boldsymbol{\sigma}$ are determined. From those the isobaric heat capacity $c_{\mathrm{p}}$, thermal expansion coefficient $\alpha_{\mathrm{p}}$, thermal conductivity along the direction normal to the solid-fluid interface, i.e. $\lambda_{\perp}$, and parallel to the solidfluid interface, i.e. $\lambda_{\|}$, as well as the shear viscosity $\eta$ are determined via post-processing. Details are given in Subsection 3.3 .

\subsection{Variants of the Simulation Setup}

As the general simulation setup is symmetrically identical to the $x z$-plane at $y=0$ and to the $y z$-plane at $x=0$, the three variants of the simulation setup are shown in Figure 2 for the first quadrant of the system $\left(x=\left[0, L_{x} / 2\right]\right.$ and $\left.y=\left[0, L_{y} / 2\right]\right)$ only. For simplicity, also the discussion in the following is conducted for the first quadrant only. However, the results that are given in Section 4 are determined from averaging over the interfacial regions of all four quadrants of the system.

Scenario I. Scenario I is shown in Figure 2 (left). Besides the mean values of $\rho, p, T$, $u, h$, and $D$, from this scenario also $c_{\mathrm{p}}, \alpha_{\mathrm{p}}$, and $\lambda_{\|}$are determined. A small temperature gradient $\partial T / \partial x=0.013$ is applied in $x$-direction by prescribing a high $\left(T_{\text {hot }}=\bar{T}+0.05\right)$ and a low temperature $\left(T_{\text {cold }}=\bar{T}-0.05\right)$ in the control volumes at the two borders of the quadrant in $x$-direction (see the red- and blue-shaded regions in Figure 2 (left)). Gaussian isokinetic thermostats are applied. The width of the temperature control volumes is $1 \sigma$ each. Along the $y$-direction the control volumes have the dimension $y=\left[2.85, L_{y} / 2\right]$.

Scenario II. In Figure 2 (middle) Scenario II is shown from which $\lambda_{\perp}$ is determined. In contrast to Scenario I, a temperature gradient $\partial T / \partial y<0.01$ is built up in the $y$ direction. It is applied by prescribing a high $\left(T_{\text {hot }}=\bar{T}+0.035\right)$ and a low temperature $\left(T_{\text {cold }}=\bar{T}-0.035\right)$ in the control volumes at the two borders of the interfacial region in $y$-direction (see red- and blue-shaded regions in Figure 2 (middle)). Gaussian isokinetic thermostats are used. The thickness of the temperature control volumes is $0.5 \sigma$ each. Along the $x$-direction the width of control volumes is equal to the width of the quadrant, i.e. $L_{x} / 2$. The bulk fluid region is also thermostatted using a Gaussian isokinetic thermostat. 




$\because \quad$ Wall

Bulk Fluid
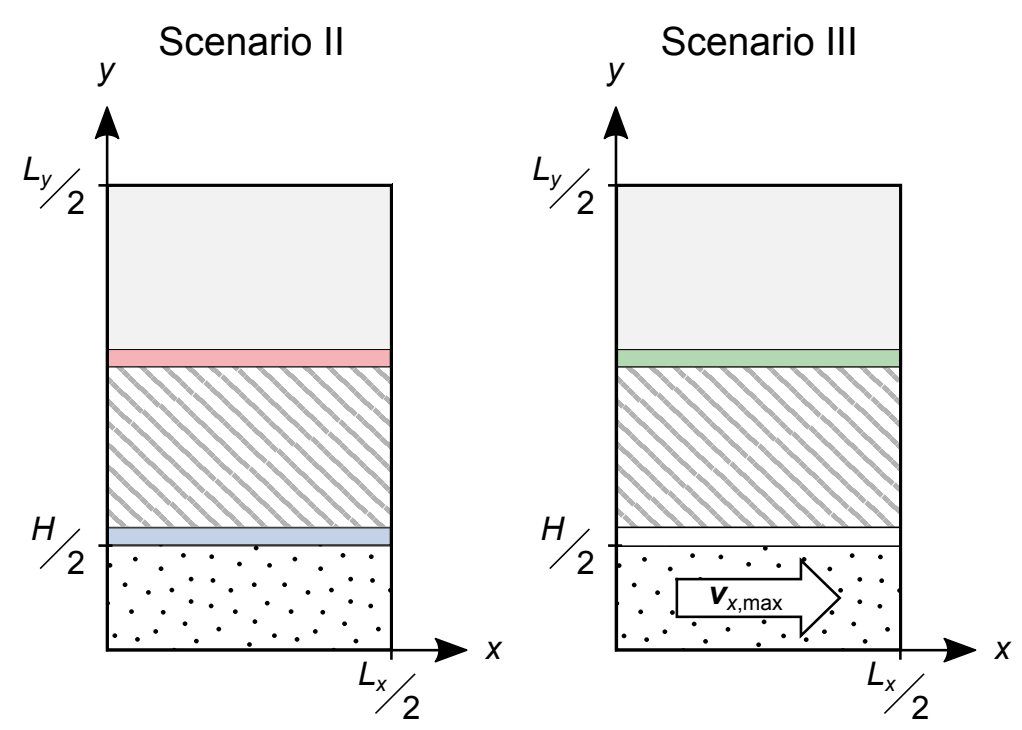

Interfacial Region

Velocity Control Volume $\left(\boldsymbol{v}_{x, 0}\right)$
Thermostat $\left(T_{\text {hot }}\right)$

Thermostat $\left(T_{\text {cold }}\right)$

Figure 2: Schemes of Scenario I (left), Scenario II (middle), and Scenario III (right) for the first quadrant $\left(x=\left[0, L_{x} / 2\right]\right.$ and $\left.y=\left[0, L_{y} / 2\right]\right)$ of the simulation setup. The wall is depicted as dotted area, the interfacial region is marked by the hatched area, and the bulk fluid region is indicated by the grey-shaded area. Left and middle: The control volumes in which the temperature is prescribed are marked in blue ( $\left.T_{\text {cold }}\right)$ and red $\left(T_{\text {hot }}\right)$. Right: The wall is moved in the $+x$-direction with the velocity $\boldsymbol{v}_{x, \max }$. The control volume in which the mean velocity $\boldsymbol{v}_{x, 0}$ is zero is marked in green.

The target temperature of the bulk fluid region is $\bar{T}$.

Scenario III. In Figure 2 (right) Scenario III is shown from which $\eta$ is determined. In contrast to both Scenario I and Scenario II, here the wall is moved in the $+x$-direction. The wall velocity is $\boldsymbol{v}_{x, \max }$. In addition, in the control volume at the upper border of the interfacial region in $y$-direction (see the green-shaded region in Figure 2 (right)) the mean velocity is $\boldsymbol{v}_{x, 0}=0 . \boldsymbol{v}_{x, \max }$ is adjusted such that the velocity gradient of the fluid in the interfacial region is $\partial \boldsymbol{v}_{x} / \partial y=0.03$. The thickness of the velocity control volume is $0.5 \sigma$. Along the $x$-direction the width of the control volume is equal to the width of the quadrant, i.e. $L_{x} / 2$. The bulk fluid region is thermostatted using a Gaussian isokinetic thermostat. The target temperature of the bulk fluid region is $\bar{T}$. 


\subsection{Fundamentals}

Temperature. The temperature is determined from the kinetic energy of the undirected thermal velocity $\boldsymbol{v}_{i, k, \text { therm }}(k=x, y, z)$ as also discussed in a previous study of our group [39]. This velocity contribution is also sometimes called the peculiar particle velocity.

$$
T=\frac{\sum_{i}^{N_{\text {bin }}} m_{i}\left(\boldsymbol{v}_{i, x, \text { therm }}^{2}+\boldsymbol{v}_{i, y, \text { therm }}^{2}+\boldsymbol{v}_{i, z, \text { therm }}^{2}\right)}{3 N_{\text {bin }} k_{\mathrm{B}}}
$$

$N_{\text {bin }}$ is the number of particles per bin, $m_{i}$ is the mass of a single particle, and $k_{\mathrm{B}}$ is the Boltzmann's constant.

Heat flux vector and stress tensor. Following the approach by Irving and Kirkwood [40], the $k$-component $(k=x, y, z)$ of the local heat flux vector $\boldsymbol{j}$ is

$$
\begin{array}{r}
\boldsymbol{j}_{k}=\frac{1}{V_{\text {bin }}\left[\sum_{i}^{N_{\text {bin }}}\left(\frac{1}{2} m_{i} \boldsymbol{v}_{i, l, \text { therm }}^{2}\right) \boldsymbol{v}_{i, k, \text { therm }}\right.}+\frac{1}{2} \sum_{i}^{N_{\text {bin }}} \sum_{j \neq i}^{N_{\text {Neighbor }}} \boldsymbol{r}_{i j, k}\left(\boldsymbol{F}_{i j, l} \boldsymbol{v}_{i, l, \text { therm }}\right) \\
\left.+\frac{1}{2} \sum_{i}^{N_{\text {bin }}} \sum_{j \neq i}^{N_{\text {Neighbor }}} \Phi\left(r_{i j}\right) \boldsymbol{v}_{i, k, \text { therm }}\right] .
\end{array}
$$

$V_{\text {bin }}$ is the bin volume. The number of neighboring particles which are interacting with the particle $i$ is $N_{\text {Neighbor }}$. The variables $\boldsymbol{v}_{i, k \text {,therm }}$ and $\boldsymbol{v}_{i, l, \text { therm }}$ correspond to the peculiar particle velocity of a particle $i$ in $k$ - and $l$-direction $(k, l=x, y, z)$, respectively. $\boldsymbol{r}_{i j, k}$ is the distance between the two particles $i$ and $j$ along the $k$-direction. $\boldsymbol{F}_{i j, l}$ and $\Phi\left(r_{i j}\right)$ are the $l$-component of the force vector and the potential energy between the same particles, respectively.

The $k l$-component of the local stress tensor in the $k l$-plane $(k, l=x, y, z)$ is also determined following the approach by Irving and Kirkwood [40]:

$$
\boldsymbol{\sigma}_{k l}=\frac{1}{V_{\text {bin }}}\left[\sum_{i}^{N_{\text {bin }}} m_{i} \boldsymbol{v}_{i, k, \text { therm }} \boldsymbol{v}_{i, l, \text { therm }}+\frac{1}{2} \sum_{i}^{N_{\text {bin }}} \sum_{j \neq i}^{N_{\text {Neighbor }}} \boldsymbol{r}_{i j, k} \boldsymbol{F}_{i j, l}\right] \text {. }
$$

Therefrom, the hydrodynamic pressure is calculated as

$$
p=\frac{1}{3} \operatorname{tr}(\boldsymbol{\sigma})
$$


Internal energy and enthalpy. The internal energy $u$ [41] is determined as

$$
u=\frac{1}{2 N_{\mathrm{bin}} M_{\mathrm{bin}}}\left[\sum_{i}^{N_{\mathrm{bin}}} m_{i} \boldsymbol{v}_{i, \text { therm }}^{2}+\sum_{i}^{N_{\mathrm{bin}}} \sum_{j \neq i}^{N_{\text {Neighbor }}} \Phi\left(r_{i j}\right)\right],
$$

Here, $M_{\text {bin }}$ corresponds to the total mass of the bin. Based on Equation 7 the enthalpy $h$ is

$$
h=u+p \frac{V_{\text {bin }}}{M_{\text {bin }}}=u+p \frac{1}{\rho_{\mathrm{m}}}
$$

where $\rho_{\mathrm{m}}$ is the mass density which is identical to the number density $\rho$ for $M=1$ as it is chosen in the present study.

Self-diffusion coefficient. Based on the mean squared displacement and under consideration of a convective flow, the $k$-component of the self-diffusion coefficient is determined as

$$
\boldsymbol{D}_{k}=\frac{1}{2 N_{\text {bin }} \Delta t}\left[\sum_{i}^{N_{\text {bin }}}\left(\boldsymbol{r}_{i, k}(t+\Delta t)-\boldsymbol{r}_{i, k}(t)-\left\langle\boldsymbol{v}_{k, \text { dir }}\right\rangle \Delta\right)^{2}\right]
$$

Equation 9 considers the distance that a particle $i$ moves along the $k$-direction within the time period $\Delta t$. Therefrom, the contribution of the convective flow $\left(\boldsymbol{v}_{k \text {, dir }}\right.$, i.e. the directed macroscopic flow velocity in $k$-direction) is subtracted. The angle brackets indicate the time-average over $\Delta t$.

As the self-diffusivity along the $y$-direction is hindered by the wall, only the components of Equation 9 parallel to the wall, i.e. $\boldsymbol{D}_{x}$ and $\boldsymbol{D}_{z}$ are considered for the determination of the self-diffusion coefficient $D$ :

$$
D=\frac{1}{2}\left(\boldsymbol{D}_{x}+\boldsymbol{D}_{z}\right)
$$

\subsection{Post-processing}

The bin-wise data of $\rho, p, T, \boldsymbol{v}_{x}, u, h, \boldsymbol{j}_{x}, \boldsymbol{\sigma}_{x y}$ and $D$ in the interfacial region are averaged (1) along the $x$-direction such that profiles in the $y$-direction are obtained, (2) along the $y$-direction such that profiles in the $x$-direction are obtained, and (3) along both directions such that mean values within the interfacial region are obtained. For the mean values data are only considered from bins that do not overlap with a temperature control volume, cf. Scenario I. Typical examples of profiles for some thermal and caloric properties 

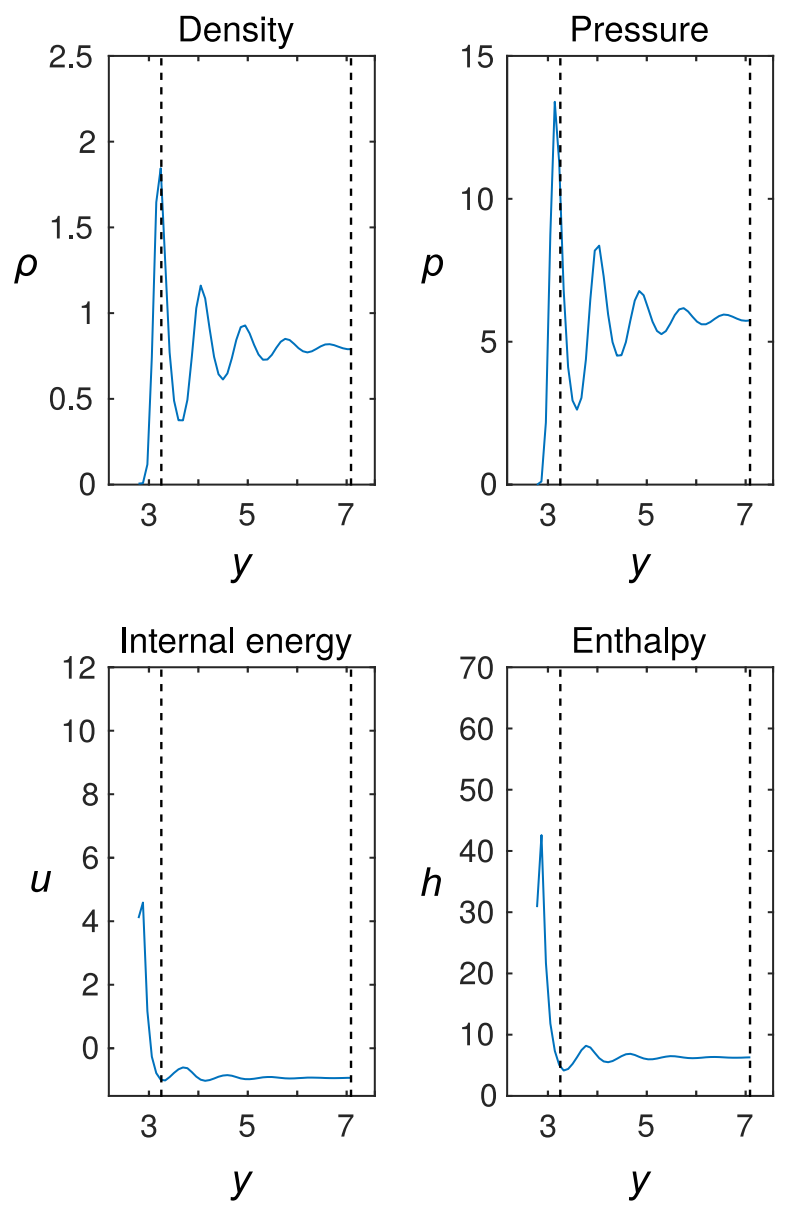

Figure 3: Overview over a selection of observables that were measured in the interfacial region from Scenario I. The bin-wise results are depicted as blue solid lines. The overall properties of the fluid are $\bar{\rho}=0.8$ and $\bar{T}=2.0$. The solid-fluid interaction is $\varepsilon_{\mathrm{sf}}=0.75$. The boundaries of the interfacial region are indicated with the vertical dashed lines.

are shown in Figure 3. Exemplary temperature gradients in $x$ - and $y$-direction as well as a velocity gradient are shown in Figure 4. They are smooth and almost perfectly linear. The gradients are so small that the mean values of $\rho, p, T, u, h$, and $D$ in the interfacial region separately determined from Scenario I, Scenario II, and Scenario III are identical within the statistical uncertainty. This indicates that the inhomogeneous structure of the fluid in the interfacial region is not significantly affected by any of the gradients and is a crucial requirement when using NEMD simulations to determine fluid properties at the solid-liquid interface [29].

The properties $c_{\mathrm{p}}, \alpha_{\mathrm{p}}, \lambda_{\perp}, \lambda_{\|}$, and $\eta$ are determined in a further post-processing step. For the data from Scenario I, linear polynomials are fitted to the profiles of $\rho(x), T(x)$ 
Scenario I

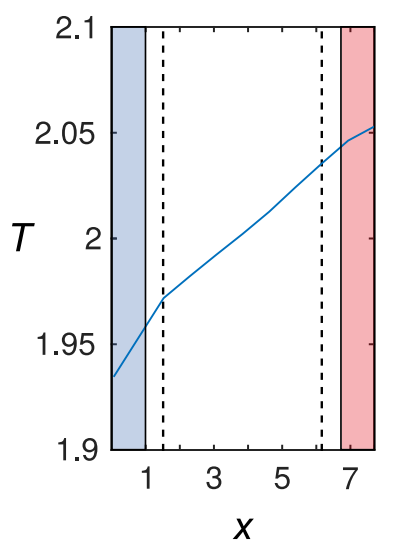

Scenario II

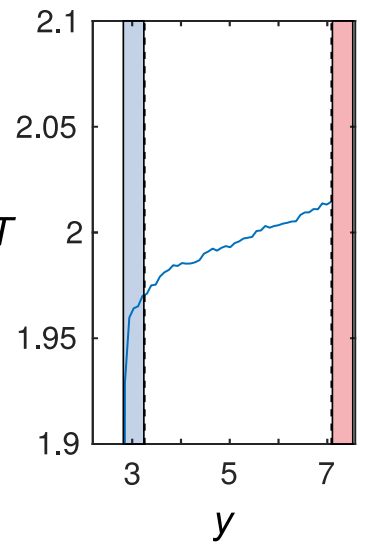

Scenario III

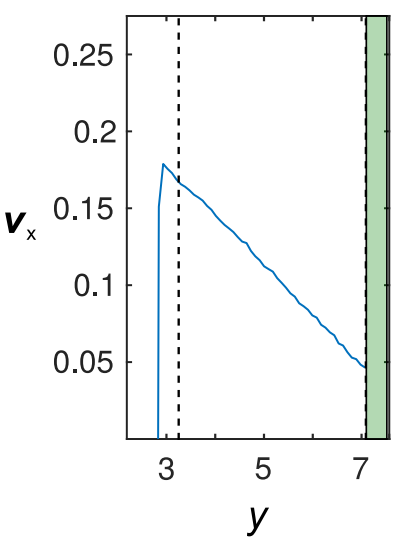

Figure 4: Profiles of the temperature and velocity gradients that were induced and measured in the interfacial region from Scenario I (left), Scenario II (middle), and Scenario III (right). The bin-wise results are depicted as blue solid lines. The overall properties of the fluid are $\bar{\rho}=0.8$ and $\bar{T}=2.0$. The solid-fluid interaction is $\varepsilon_{\mathrm{sf}}=0.75$. The boundaries of the interfacial region are indicated with the vertical dashed lines. The temperature and velocity control volumes in the $y z$-plane (left) and $x z$-plane (middle and right) are indicated by the shaded areas from which the colors follow the legend of Figure 2

and $h(x)$. Therefrom, the numbers for $c_{\mathrm{p}}, \alpha_{\mathrm{p}}$, and $\lambda_{\|}$are determined as

$$
\begin{gathered}
c_{\mathrm{p}}=\frac{\partial h(x)}{\partial T(x)}=\frac{\partial h(x)}{\partial x}\left(\frac{\partial T(x)}{\partial x}\right)^{-1}, \\
\alpha_{\mathrm{p}}=-\frac{\partial(\ln \rho(x))}{\partial T(x)}=-\frac{\partial(\ln \rho(x))}{\partial x}\left(\frac{\partial T(x)}{\partial x}\right)^{-1}, \\
\lambda_{\|}=-\boldsymbol{j}_{x}\left(\frac{\partial T(x)}{\partial x}\right)^{-1},
\end{gathered}
$$

where $\boldsymbol{j}_{x}$ is the mean value of the heat flux in the $x$-direction determined from Scenario I.

For Scenario II, also a linear polynomial is fitted to the profile of $T(y)$ such that $\lambda_{\perp}$ is determined as

$$
\lambda_{\perp}=-\tilde{\boldsymbol{j}}_{y}\left(\frac{\partial T(y)}{\partial x}\right)^{-1}
$$

For Scenario II, the heat flux $\tilde{\boldsymbol{j}}_{y}$ between the two control volumes, i.e. the heat flux in the interfacial region, is determined as

$$
\tilde{\boldsymbol{j}}_{y}=\frac{\Delta E_{k i n}}{t L_{x} L_{z}}=\frac{E_{k i n, T_{\mathrm{hot}}}+E_{k i n, T_{\text {bulk }}}}{t L_{x} L_{z}}
$$


where $\Delta E_{k i n}$ is the kinetic energy that is put into the interfacial region during the simulation run time $t$. It consists of a positive contribution $E_{k i n, T_{\text {hot }}}$, i.e. the kinetic energy that is put into the control volume where $T_{\text {hot }}$ is applied, and a negative contribution $E_{k i n, T_{\text {bulk }}}$, i.e. the kinetic energy that is removed by the thermostat in the bulk fluid region.

Finally, for Scenario III, a linear polynomial is fitted to the profile of $\boldsymbol{v}_{x}(y)$. As the velocity gradient is small and almost perfectly linear (cf. Figure 4 (right)), the linear Newton's law can be applied [42] and the viscosity is determined as

$$
\eta=-\boldsymbol{\sigma}_{x y}\left(\frac{\partial \boldsymbol{v}_{x}(y)}{\partial y}\right)^{-1}
$$

Here, $\boldsymbol{\sigma}_{x y}$ is the mean value of the $x y$-component of the shear stresses in the interfacial region determined from Scenario III.

\subsection{Simulation Details}

The simulation details as described in the following apply to all aforementioned simulation scenarios. A simulation run consists of 2,000,000 time steps in which a steady state is established, i.e. stable temperature or velocity gradients are built up, respectively. Subsequently, a production run of 4,000,000 time steps is conducted. Block-averaging is applied over 200,000 time steps each (cf. Reference [35]). Thus, all properties studied in the present work are determined separately for each block. The mean standard deviation as given in the following then is the mean standard deviation of the block-averages. The equations of motion were solved using a leapfrog integrator with the time step $\Delta \tau=0.002$. The number of particles in the simulation box as well as the volume of the simulation box were constant.

The simulations were carried out with a version of the MD code ls1 mardyn [43] that was substantially extended and from which the extensions that are relevant for the present study are described in recent works of our group [39, 44].

\section{Results and Discussion}

\subsection{Thermal and Caloric Properties}

Figure 5 shows the average values of the properties $p, \rho, u, h, c_{\mathrm{p}}$, and $\alpha_{\mathrm{p}}$ of the fluid

in the interfacial region as a function of $\varepsilon_{\mathrm{sf}}$ and for three temperatures: $\bar{T}=0.7$ (blue 
squares), $\bar{T}=0.8$ (green diamonds), and $\bar{T}=2.0$ (red circles). The data are compared to the bulk properties of the LJTS fluid at the state $(\bar{\rho}, \bar{T})$ which were determined using the equation of state (EOS) of Thol et al. [45]. The numerical results as well as the mean standard deviations of the thermal and caloric properties are given in the Supporting Information.

For a given $\bar{\rho}$, all thermal and caloric properties depend on $\varepsilon_{\mathrm{sf}}$ and $\bar{T}$. However, the results are close to the bulk values with deviations which are generally below $15 \%$, cf. Figure 5. This is also shown with a deviation plot in Figure S5 in the Supporting Information. The trend of the data at high temperature $(\bar{T}=2.0)$ differs from those at low temperatures $(\bar{T}=\{0.7,0.8\})$. Especially $\rho$ and $c_{\mathrm{p}}$ show a different dependence on $\varepsilon_{\mathrm{sf}}$ at low and high temperatures.

$p, u$, and $h$ generally decrease with increasing $\varepsilon_{\text {sf }}$. This is mainly due to the weighty contribution from the first layer which is closest to the solid-fluid interface and is shown in detail in the Figures S1 to S4 in the Supporting Information. Except of $u$, the fluid properties in the interfacial region can be both larger or smaller than their bulk values. The results indicate that they are closest to the bulk values when $\bar{T} \approx \varepsilon_{\mathrm{sf}}$.

A non-uniform behavior is found for $\rho, c_{\mathrm{p}}$, and $\alpha_{\mathrm{p}} \cdot \rho$ is larger than the bulk density when $\bar{T}>\varepsilon_{\text {sf }}$ and smaller when $\bar{T} \lesssim \varepsilon_{\text {sf }}$. With increasing $\varepsilon_{\mathrm{sf}}, c_{\mathrm{p}}$ increases when the fluid temperature is low $(\bar{T}=\{0.7,0.8\})$ and decreases when the fluid temperature is high $(\bar{T}=2.0) . \alpha_{\mathrm{p}}$ generally increases with increasing $\varepsilon_{\mathrm{sf}}$. It can be both larger or smaller than the bulk value. The results from the present work indicate that it is larger when $\bar{T}>\varepsilon_{\text {sf }}$ and smaller when $\bar{T} \lesssim \varepsilon_{\mathrm{sf}}$.

\subsection{Transport Properties}

Figure 6 shows the average values of $\lambda_{\perp}, \lambda_{\|}, \eta$, and $D$ of the fluid in the interfacial region as a function of $\varepsilon_{\mathrm{sf}}$ and for three temperatures: $\bar{T}=0.7$ (blue squares), $\bar{T}=0.8$ (green diamonds), and $\bar{T}=2.0$ (red circles). The data are compared to the bulk properties of the LJTS fluid at the state $(\bar{\rho}, \bar{T})$ which were determined using the correlations from a previous work of our group [46]. The numerical results as well as the mean standard deviations of the transport properties are given in the Supporting Information.

Similar to the thermal and caloric properties discussed above, for a given $\bar{\rho}$ also the 

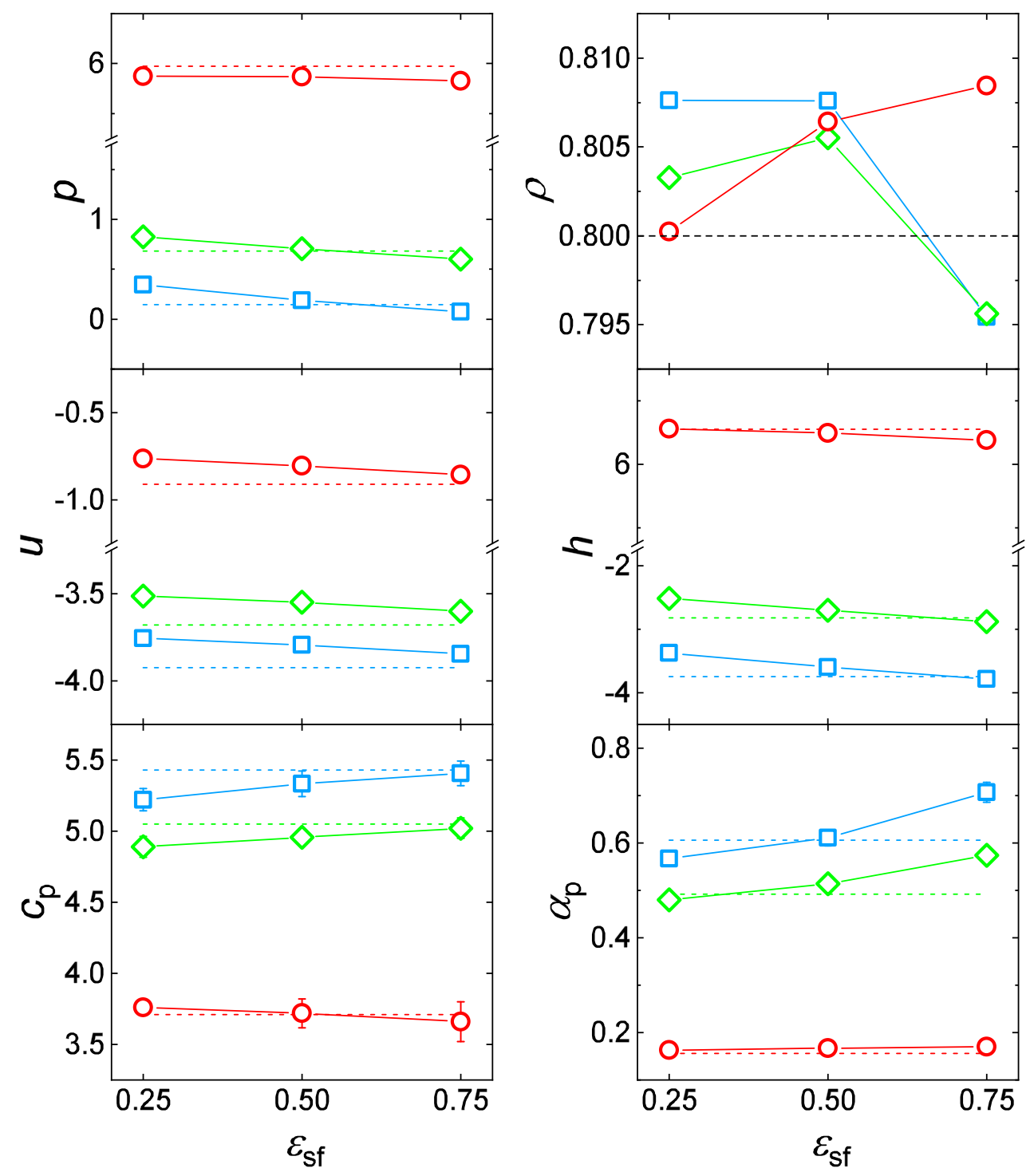

Figure 5: Thermal and caloric properties of the LJTS fluid in the interfacial region. Data are shown for three temperatures: $\bar{T}=0.7$ (blue squares), $\bar{T}=0.8$ (green diamonds), and $\bar{T}=2.0$ (red circles). The values of the corresponding bulk properties of the LJTS fluid are indicated with dashed lines in the same color. They were determined with the EOS of Thol et al. 45. The estimated uncertainties of the simulation data are indicated by the errors bars where they exceed the symbol size. The connecting lines have the purpose to guide the eyes only. 

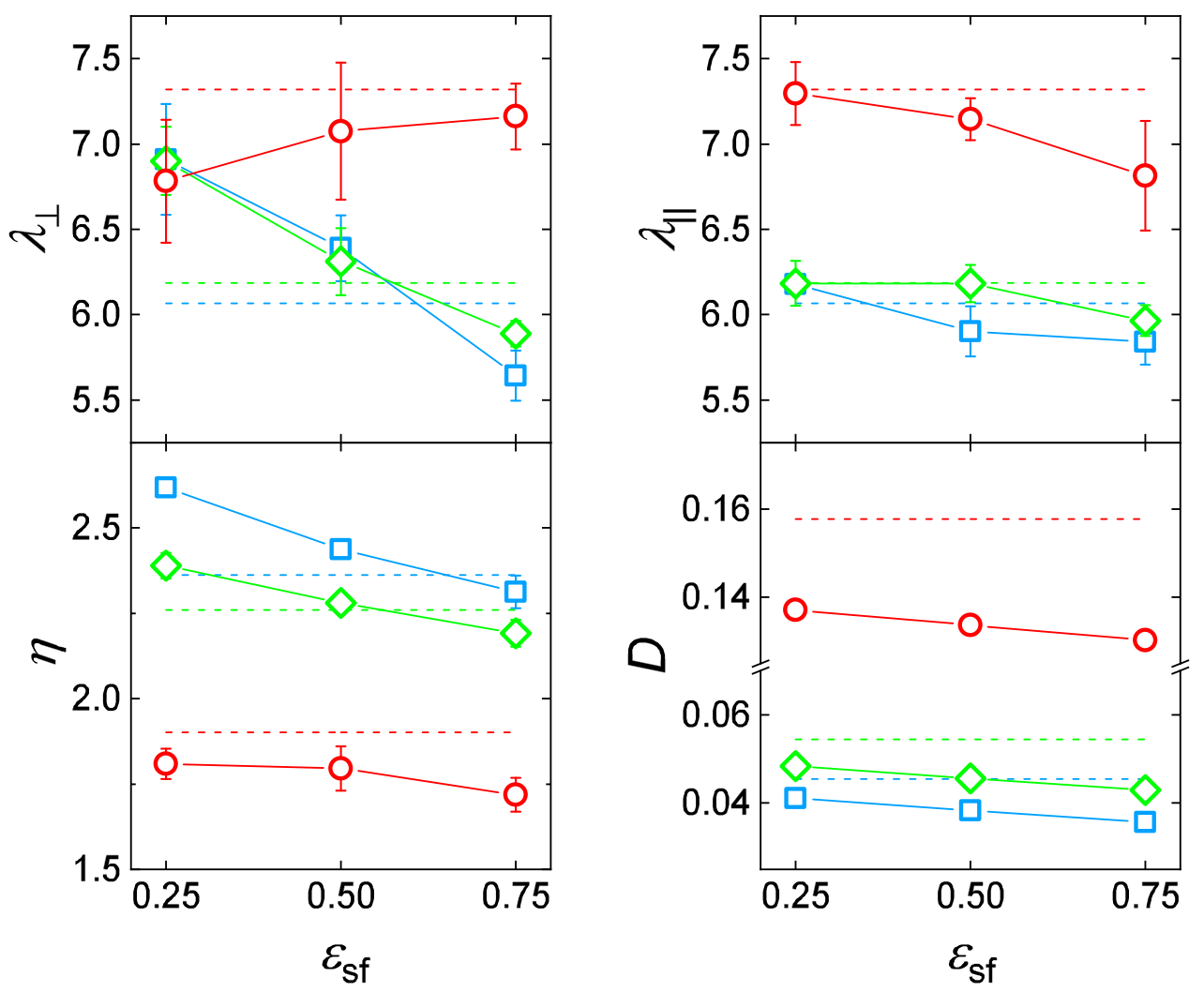

Figure 6: Transport properties of the LJTS fluid in the interfacial region. Data are shown for three temperatures: $\bar{T}=0.7$ (blue squares), $\bar{T}=0.8$ (green diamonds), and $\bar{T}=2.0$ (red circles). The values of the corresponding bulk properties of the LJTS fluid are indicated with dashed lines in the same color. They were determined with the correlations from a previous work of our group [46]. The estimated uncertainties of the simulation data are indicated by the errors bars where they exceed the symbol size. The connecting lines have the purpose to guide the eyes only. 
transport properties depend on $\varepsilon_{\mathrm{sf}}$ and $\bar{T}$. The data are again close to the bulk values with deviations which are generally below $15 \%$, cf. Figure 6 . This is also shown with a deviation plot in Figure S6 in the Supporting Information. The trend of the data at high temperature $(\bar{T}=2.0)$ differs from those at low temperatures $(\bar{T}=\{0.7,0.8\})$. Especially the data on $\lambda_{\perp}$ show a contrary dependence on $\varepsilon_{\mathrm{sf}}$. Except of $D$ which is always below the bulk value, the other transport properties can be both larger or smaller than the bulk value. In general the data decrease with increasing $\varepsilon_{\mathrm{sf}}$. This is partially induced by the decreasing pressure in the interfacial region, cf. Figure 5. The only exception from this behavior is observed for $\lambda_{\perp}$ which increases with increasing $\varepsilon_{\text {sf }}$.

These findings on the behavior of transport properties in the interfacial region are compared with general trends as they are reported in the literature. However,the results from the literature were mainly determined for fluids under strong confinement where the fluid properties were determined as mean values from the entire nanochannel. Moreover, the values of $\varepsilon_{\mathrm{sf}}$ used in studies of the literature were mainly larger compared to those used in the present study. (1) Concerning $\lambda_{\perp}$ both the results from the present work and the results from the literature show that $\lambda_{\perp}$ is always smaller than the bulk value when $\bar{T} \approx \varepsilon_{\text {sf }}[23,27,47]$. Moreover, $\lambda_{\perp}$ is always smaller than the bulk value and decreases with decreasing $\varepsilon_{\text {sf }}$ when $\bar{T} \gtrsim \varepsilon_{\mathrm{f}}\left[28\right.$, 47]. (2) $\lambda_{\|}$is smaller than the bulk value when $\bar{T} \approx \varepsilon_{\mathrm{sf}}$ [23, 26, 27, 48]. Moreover, for $\bar{T} \lesssim 0.8$ and large values of $\varepsilon_{\mathrm{sf}}, \lambda_{\|}$is always larger than $\lambda_{\perp}$ [49, 50]. (3) Compared to the thermal conductivities, the viscosity is less affected by the structural inhomogeneities in the interfacial region [23, 29, 30, 51]. Thus, also the effect

of $\varepsilon_{\mathrm{sf}}$ on $\eta$ is lower than on other transport properties [23, 29, 52]. (4) $D$ is significantly lower than its bulk value [8, 15, 22, 24] and decreases with increasing $\varepsilon_{\mathrm{sf}}[22$.

In some cases, however, in the literature also trends were observed that differ from the findings in the present study: some authors [25, 52, 53] found that $\lambda_{\|}$increases with increasing $\varepsilon_{\mathrm{sf}}$ and one working group [53] stated that $\lambda_{\|}$is always larger than the bulk value.

\section{Conclusion}

In the present study, non-equilibrium molecular dynamics (NEMD) simulations were used to determine thermal, caloric, and transport properties of a fluid in the highly struc- 
tured and inhomogeneous region close to a wall. The interfacial region studied in the present work is intentionally not affected by a frustration of ordering. Thus, when the overall density of the fluid is constant, the structure of the fluid is solely influenced by the solid-fluid interaction energy and the temperature of the fluid. The properties that were determined as averaged values in the interfacial region are: pressure $p$, density $\rho$, internal energy $u$, enthalpy $h$, isobaric heat capacity $c_{\mathrm{p}}$, thermal expansion coefficient $\alpha_{\mathrm{p}}$, thermal conductivity $\lambda$, shear viscosity $\eta$, and self-diffusion coefficient $D$. Concerning the thermal conductivity, $\lambda_{\perp}$, i.e. perpendicular to the solid-fluid interface, and $\lambda_{\|}$, i.e. parallel to the solid-fluid interface were studied separately.

All interactions were modeled using the Lennard-Jones potential truncated and shifted at a cut-off radius of $r_{\mathrm{c}}=2.5$ (LJTS). The overall fluid density was kept constant $(\bar{\rho}=0.8)$ and the overall fluid temperature $\bar{T}=\{0.7,0.8,2.0\}$ as well as the solid-fluid interaction $\varepsilon_{\mathrm{sf}}=\{0.25,0.50,0.75\}$ were varied. Influences on the aforementioned fluid properties were studied. The simulation results were compared to the bulk properties of the LJTS fluid at the corresponding states $(\bar{\rho}, \bar{T})$.

In general the findings from the present study indicate that for a given $\bar{\rho}$, all fluid properties studied in the present work are affected by $\varepsilon_{\text {sf }}$ and $\bar{T}$. However, the deviations from the bulk properties are generally below $15 \%$ for the physically relevant range of values on $\varepsilon_{\mathrm{sf}}$ studied in the present work. The deviations from the bulk behavior are caused by the interdependence between $\varepsilon_{\text {sf }}$ and $\bar{T}$. Trends of the behavior found in the present study were compared to those reported in the literature and mainly concordances were found.

The results from the present work give an insight in how structural inhomogeneities, that are solely induced by the solid-fluid interaction in the present work, as well as the interplay with the fluid temperature affect various fluid properties. However, a deep insight into the physical background still lacks. Therefore, it would be interesting for a future work to apply the density functional theory to the data from the present work to shed more light on the reasons of the behavior reported here.

\section{Supplementary Material}

See the Supporting Information for the numerical results and the mean standard deviations of all properties for each state point studied in the present work. There, also the 
percentage deviation of the fluid properties in the interfacial region from the corresponding bulk values is shown in deviation plots. In addition the fluid properties are compared to the bulk values at the pressures as they are determined from the interfacial region.

\section{Conflicts of interest}

There are no conflicts to declare.

\section{Acknowledgement}

The project was funded by the Deutsche Forschungsgemeinschaft (DFG, German Research Foundation) - 252408385 - IRTG 2057 Physical Modeling for Virtual Manufacturing Systems and Processes as well as by the Federal Ministry of Education and Research (BMBF, Germany) - 01IH16008 - Task-based load balancing and auto-tuning in particle simulations (TaLPas). The simulations were carried out on the elwe at Regional University Computing Center Kaiserslautern (RHRK) under the grant TUKL-TLMV as well as on the HAZELHEN at the Supercomputing Centre Stuttgart (HLRS) under the grant 'Molecular Modelling of Hydrogen Bonding Fluids' (MMHBF2). The present research was conducted under the auspices of the Boltzmann-Zuse Society for Computational Molecular Engineering (BZS). 
[1] J. N. Israelachvili, Intermolecular and Surface Forces, Vol. 53, Academic Press, New York, 2011. doi:10.1016/C2009-0-21560-1.

[2] D. G. Cahill, P. V. Braun, G. Chen, D. R. Clarke, S. Fan, K. E. Goodson, P. Keblinski, W. P. King, G. D. Mahan, A. Majumdar, H. J. Maris, S. R. Phillpot, E. Pop, L. Shi, Nanoscale thermal transport. II. 2003-2012, Applied Physics Reviews 1 (1) (2014) 011305. doi:10.1063/1.4832615.

[3] S. Razavi, J. Koplik, I. Kretzschmar, Molecular Dynamics Simulations: Insight into Molecular Phenomena at Interfaces, Langmuir 30 (38) (2014) 11272-11283. doi: 10.1021/la500376z.

URL http: //pubs .acs . org/doi/10.1021/la500376z

[4] O. Björneholm, M. H. Hansen, A. Hodgson, L.-M. Liu, D. T. Limmer, A. Michaelides, P. Pedevilla, J. Rossmeisl, H. Shen, G. Tocci, E. Tyrode, M.-M. Walz, J. Werner, H. Bluhm, Water at Interfaces, Chemical Reviews 116 (13) (2016) 7698-7726. doi: 10.1021/acs. chemrev.6b00045.

URL http://pubs .acs .org/doi/10.1021/acs . chemrev.6b00045

[5] J. J. Magda, M. Tirrell, H. T. Davis, Molecular dynamics of narrow, liquid-filled pores, The Journal of Chemical Physics 83 (4) (1985) 1888-1901. doi:10.1063/1.449375.

[6] L. A. Pozhar, Structure and dynamics of nanofluids: Theory and simulations to calculate viscosity, Physical Review E 61 (2) (2000) 1432-1446. doi:10.1103/PhysRevE.61.1432.

URL http://link.aps.org/abstract/PRE/v61/p1432https://link.aps.org/ doi/10.1103/PhysRevE.61.1432

[7] P. A. Thompson, M. O. Robbins, Shear flow near solids: Epitaxial order and flow boundary conditions, Physical Review A 41 (12) (1990) 6830-6837. doi:10.1103/ PhysRevA.41.6830.

[8] H. Bock, K. E. Gubbins, M. Schoen, Anisotropic Self-Diffusion in Nanofluidic Structures, The Journal of Physical Chemistry C 111 (43) (2007) 15493-15504. doi: 
10.1021/jp071861y.

URL http://pubs .acs .org/doi/abs/10.1021/jp071861y

[9] S. A. Somers, H. T. Davis, Microscopic dynamics of fluids confined between smooth and atomically structured solid surfaces, The Journal of Chemical Physics 96 (7) (1992) 5389-5407. doi:10.1063/1.462724.

URL http://aip.scitation.org/doi/10.1063/1.462724

[10] R. G. Horn, J. N. Israelachvili, Direct measurement of structural forces between two surfaces in a nonpolar liquid, The Journal of Chemical Physics 75 (3) (1981) 14001411. doi:10.1063/1.442146.

[11] F. Sofos, T. E. Karakasidis, A. Liakopoulos, Fluid Flow at the Nanoscale: How Fluid Properties Deviate from the Bulk, Nanoscience and Nanotechnology Letters 5 (4) (2013) 457-460. doi:10.1166/nnl.2013.1555

URL http://openurl.ingenta.com/content/xref?genre=article $\{\&\}$ issn= $1941-4900\{\&\}$ volume $=5\{\&\}$ issue $=4\{\&\}$ spage $=457$

[12] J. Gao, W. D. Luedtke, U. Landman, Structure and solvation forces in confined films: Linear and branched alkanes, The Journal of Chemical Physics 106 (10) (1997) 43094318. doi:10.1063/1.473132.

URL http://aip.scitation.org/doi/10.1063/1.473132

[13] M. L. Gee, P. M. McGuiggan, J. N. Israelachvili, A. M. Homola, Liquid to solidlike transitions of molecularly thin films under shear, The Journal of Chemical Physics 93 (3) (1990) 1895-1906. doi:10.1063/1.459067.

URL http://aip.scitation.org/doi/10.1063/1.459067

[14] I. Bitsanis, J. J. Magda, M. Tirrell, H. T. Davis, Molecular dynamics of flow in micropores, The Journal of Chemical Physics 87 (3) (1987) 1733-1750. doi:10. $1063 / 1.453240$.

URL http://aip.scitation.org/doi/10.1063/1.453240

[15] K. Tankeshwar, S. Srivastava, Dynamical model for restricted diffusion in nano- 
channels, Nanotechnology 18 (48) (2007) 485714. doi:10.1088/0957-4484/18/48/ 485714.

[16] R. Devi, J. Sood, S. Srivastava, K. Tankeshwar, Diffusion of fluid confined to nanotube with rectangular cross section, Microfluidics and Nanofluidics 9 (4-5) (2010) 737-742. doi:10.1007/s10404-010-0587-2.

[17] R. Devi, S. Srivastava, K. Tankeshwar, The role of fluid-wall interactions on confined liquid diffusion using Mori theory, The Journal of Chemical Physics 143 (2) (2015) 024506. doi:10.1063/1.4926619.

URL http://aip.scitation.org/doi/10.1063/1.4926619

[18] V. Y. Rudyak, A. A. Belkin, Self-diffusion and viscosity coefficient of fluids in nanochannels, in: 3rd Micro and Nano Flows Conference, no. August, 2011, pp. $22-24$.

URL http://v-scheiner.brunel.ac.uk/handle/2438/6732

[19] J. Gao, W. D. Luedtke, U. Landman, Layering transitions and dynamics of confined liquid films, Physical Review Letters 79 (4) (1997) 705-708. doi:10.1103/ PhysRevLett.79.705.

[20] A. Giannakopoulos, F. Sofos, T. Karakasidis, A. Liakopoulos, Unified description of size effects of transport properties of liquids flowing in nanochannels, International Journal of Heat and Mass Transfer 55 (19-20) (2012) 5087-5092. doi:10.1016/j.ijheatmasstransfer.2012.05.008.

URL http://dx.doi.org/10.1016/j.ijheatmasstransfer.2012.05.008https: //linkinghub.elsevier.com/retrieve/pii/S0017931012003213

[21] T.-D. Li, J. Gao, R. Szoszkiewicz, U. Landman, E. Riedo, Structured and viscous water in subnanometer gaps, Physical Review B 75 (11) (2007) 115415. doi:10. 1103/PhysRevB.75.115415.

URL https://link.aps .org/doi/10.1103/PhysRevB.75.115415

[22] C. Zhang, Y. Chen, L. Yang, M. Shi, Self-diffusion for Lennard-Jones fluid confined in a nanoscale space, International Journal of Heat and Mass Transfer 54 (21-22) (2011) 
4770-4773. doi:10.1016/j.ijheatmasstransfer.2011.05.032.

URL http://dx.doi.org/10.1016/j.ijheatmasstransfer.2011.05.032

[23] F. Sofos, T. Karakasidis, A. Liakopoulos, Transport properties of liquid argon in krypton nanochannels: Anisotropy and non-homogeneity introduced by the solid walls, International Journal of Heat and Mass Transfer 52 (3-4) (2009) 735-743. doi:10.1016/j.ijheatmasstransfer.2008.07.022.

URL http://dx.doi.org/10.1016/j.ijheatmasstransfer.2008.07.022http:// linkinghub.elsevier.com/retrieve/pii/S0017931008004407

[24] Y. Liu, Q. Wang, T. Wu, L. Zhang, Fluid structure and transport properties of water inside carbon nanotubes, The Journal of Chemical Physics 123 (23) (2005) 234701. doi:10.1063/1.2131070.

[25] M. Frank, D. Drikakis, Thermodynamics at Solid-Liquid Interfaces, Entropy 20 (5) (2018) 362. doi:10.3390/e20050362.

URL http://www.mdpi.com/1099-4300/20/5/362

[26] K. Hyżorek, K. V. Tretiakov, Thermal conductivity of liquid argon in nanochannels from molecular dynamics simulations, The Journal of Chemical Physics 144 (19) (2016) 194507. doi:10.1063/1.4949270.

URL http://scitation.aip.org/content/aip/journal/jcp/144/19/10.1063/ 1.4949270http://aip.scitation.org/doi/10.1063/1.4949270

[27] S. Murad, P. Ravi, J. G. Powles, Anisotropic thermal conductivity of a fluid in a system of microscopic slit pores, Physical Review E 48 (5) (1993) 4110-4112. doi: 10.1103/PhysRevE.48.4110.

URL https://link.aps .org/doi/10.1103/PhysRevE.48.4110

[28] Q.-X. Liu, P.-X. Jiang, H. Xiang, Molecular dynamics simulation of thermal conductivity of an argon liquid layer confined in nanospace, Molecular Simulation 36 (13) (2010) 1080-1085. doi:10.1080/08927022.2010.504773.

URL http://www . tandfonline.com/doi/abs/10.1080/08927022.2010.504773 
[29] J. Petravic, P. Harrowell, Equilibrium calculations of viscosity and thermal conductivity across a solid-liquid interface using boundary fluctuations., The Journal of chemical physics 128 (19) (2008) 194710. doi:10.1063/1.2911924.

[30] M. Neek-Amal, F. M. Peeters, I. V. Grigorieva, A. K. Geim, Commensurability Effects in Viscosity of Nanoconfined Water, ACS Nano 10 (3) (2016) 3685-3692. doi:10.1021/acsnano.6b00187.

URL https://pubs.acs.org/doi/abs/10.1021/acsnano.6b00187http:

//pubs.acs.org/doi/10.1021/acsnano.6b00187

[31] I. Goyal, S. Srivastava, K. Tankeshwar, Controlling Diffusion by Varying Width of Layers in Nano Channel, Nano-Micro Letters 4 (3) (2012) 154-157. doi:10.1007/ BF03353706.

URL http://link. springer. com/10.1007/BF03353706

[32] J. P. Ewen, D. M. Heyes, D. Dini, Advances in nonequilibrium molecular dynamics simulations of lubricants and additives, Friction 6 (4) (2018) 349-386. doi:10.1007/ s40544-018-0207-9.

URL http: //link . springer .com/10.1007/s40544-018-0207-9

[33] J. Gao, W. D. Luedtke, U. Landman, Structures, solvation forces and shear of molecular films in a rough nano-confinement, Tribology Letters 9 (2000) 3-13. doi:10.1023/A:1018840023845.

[34] S. Becker, H. M. Urbassek, M. Horsch, H. Hasse, Contact Angle of Sessile Drops in Lennard-Jones Systems, Langmuir 30 (45) (2014) 13606-13614. doi:10.1021/ $1 \mathrm{a} 503974 \mathrm{z}$.

URL http://pubs . acs . org/doi/10.1021/la503974z

[35] M. P. Allen, D. J. Tildesley, Computer simulation of liquids, Oxford Science Publications, New York, 1989.

[36] S. Becker, M. Kohns, H. M. Urbassek, M. Horsch, H. Hasse, Static and Dynamic Wetting Behavior of Drops on Impregnated Structured Walls by Molecular Dynamics Simulation, The Journal of Physical Chemistry C 121 (23) (2017) 12669-12683. doi: 
10.1021/acs.jpcc.6b12741.

URL http://pubs . acs . org/doi/10.1021/acs . jpcc .6b12741

[37] M. Holmboe, I. C. Bourg, Molecular Dynamics Simulations of Water and Sodium Diffusion in Smectite Interlayer Nanopores as a Function of Pore Size and Temperature, The Journal of Physical Chemistry C 118 (2) (2014) 1001-1013. doi: $10.1021 / \mathrm{jp} 408884 \mathrm{~g}$.

URL http://pubs .acs.org/doi/10.1021/jp408884g

[38] P. Simonnin, B. Noetinger, C. Nieto-Draghi, V. Marry, B. Rotenberg, Diffusion under Confinement: Hydrodynamic Finite-Size Effects in Simulation, Journal of Chemical Theory and Computation 13 (6) (2017) 2881-2889. doi:10.1021/acs .jctc.7b00342. URL http://pubs.acs.org/doi/10.1021/acs. jctc.7b00342

[39] M. P. Lautenschlaeger, M. Horsch, H. Hasse, Simultaneous determination of thermal conductivity and shear viscosity using two-gradient non-equilibrium molecular dynamics simulations, Molecular Physics 117 (2) (2019) 189-199. doi:10.1080/ 00268976.2018 .1504134 .

[40] J. Irving, J. Kirkwood, The Statistical Mechanics Theory of Transport Processes. IV. The Equations of Hydrodynamics, Journal of Chemical Physics 18 (06) (1950) $817-829$.

[41] D. J. Evans, G. P. Morriss, Statistical Mechanics of Nonequilibrium Liquids, no. 1, ANU E Press, Canberra, 2007. doi:10.1017/CB09780511535307.

[42] M. Morciano, M. Fasano, A. Nold, C. Braga, P. Yatsyshin, D. N. Sibley, B. D. Goddard, E. Chiavazzo, P. Asinari, S. Kalliadasis, Nonequilibrium molecular dynamics simulations of nanoconfined fluids at solid-liquid interfaces, The Journal of Chemical Physics 146 (24) (2017) 244507. doi:10.1063/1.4986904.

URL http://aip.scitation.org/doi/10.1063/1.4986904

[43] C. Niethammer, S. Becker, M. Bernreuther, M. Buchholz, W. Eckhardt, A. Heinecke, S. Werth, H.-J. Bungartz, C. W. Glass, H. Hasse, J. Vrabec, M. Horsch, 
Is1 mardyn : The Massively Parallel Molecular Dynamics Code for Large Systems, Journal of Chemical Theory and Computation 10 (10) (2014) 4455-4464. doi:10.1021/ct500169q.

URL http://pubs.acs.org/doi/abs/10.1021/ct500169q

[44] M. P. Lautenschlaeger, H. Hasse, Thermal and Caloric Properties of Fluids from NonEquilibrium Molecular Dynamics Simulations Using the Two-Gradient Method, The Journal of Chemical Physics 149 (24) (2018) 244106. doi:10.1063/1.5063985.

[45] M. Thol, G. Rutkai, R. Span, J. Vrabec, R. Lustig, Equation of State for the LennardJones Truncated and Shifted Model Fluid, International Journal of Thermophysics 36 (1) (2015) 25-43. doi:10.1007/s10765-014-1764-4.

URL http://link. springer.com/10.1007/s10765-014-1764-4

[46] M. P. Lautenschlaeger, H. Hasse, Transport Properties of the Lennard-Jones Truncated and Shifted Model Fluid from Non-Equilibrium Molecular Dynamics Calculations, Fluid Phase Equilibria 482 (2019) 38 - 47. doi:https://doi.org/10.1016/ j.fluid.2018.10.019.

[47] J. Petravic, P. Harrowell, Spatial Dependence of Viscosity and Thermal Conductivity through a Planar Interface, The Journal of Physical Chemistry B 113 (7) (2009) 2059-2065. doi:10.1021/jp807254b.

URL http://www.ncbi.nlm.nih.gov/pubmed/19199687http://pubs.acs.org/ doi/abs/10.1021/jp807254b

[48] R. Hannaoui, G. Galliéro, D. Ameur, C. Boned, Molecular dynamics simulations of heat and mass transport properties of a simple binary mixture in micro/meso-pores, Chemical Physics 389 (1-3) (2011) 53-57. doi:10.1016/j.chemphys.2011.07.029. URL http://dx.doi.org/10.1016/j .chemphys .2011.07.029

[49] C. Sun, W.-Q. Lu, B. Bai, J. Liu, Anomalous enhancement in thermal conductivity of nanofluid induced by solid walls in a nanochannel, Applied Thermal Engineering 31 (17-18) (2011) 3799-3805. doi:10.1016/j.applthermaleng.2011.07.021. 
URL http://dx.doi.org/10.1016/j.applthermaleng.2011.07.021https:

//linkinghub.elsevier.com/retrieve/pii/S1359431111003784

[50] C. Sun, W.-Q. Lu, B. Bai, J. Liu, Transport properties of Ar-Kr binary mixture in nanochannel Poiseuille flow, International Journal of Heat and Mass Transfer 55 (5-6) (2012) 1732-1740. doi:10.1016/j.ijheatmasstransfer.2011.11.028.

URL http://dx.doi.org/10.1016/j.ijheatmasstransfer.2011.11.028https: //linkinghub.elsevier.com/retrieve/pii/S001793101100665X

[51] L. Bocquet, E. Charlaix, Nanofluidics, from bulk to interfaces, Chemical Society Reviews 39 (3) (2010) 1073-1095. doi:10.1039/b909366b.

URL http://xlink.rsc.org/?DOI=B909366B

[52] Y. Liu, Q. Wang, L. Zhang, T. Wu, Dynamics and Density Profile of Water in Nanotubes as One-Dimensional Fluid, Langmuir 21 (25) (2005) 12025-12030. doi:10.1021/la0517181.

URL http://pubs .acs.org/doi/abs/10.1021/la0517181

[53] M. Frank, D. Drikakis, Solid-like heat transfer in confined liquids, Microfluidics and Nanofluidics 21 (9) (2017) 148. doi:10.1007/s10404-017-1980-x.

URL http://link.springer.com/10.1007/s10404-017-1980-x 